\title{
Modeling of evolution of the rotational axis of "hot Jupiter" planets under tidal perturbations
}

\author{
Irina Kitiashvili \\ Kazan state university, Dept. of Astronomy \\ 420008, Kremlevskaya str., 18, Kazan, Russia \\ email: gabbiano@mail.ru
}

\begin{abstract}
In this report, we present results of analytical and numerical calculations of evolution the axis of rotation of planets moving at very close orbits. We consider the evolution of the axis of rotation caused by tidal perturbations of a parent star and obtain estimates of the principal moment of inertia and the dynamical flattening for nine exoplanets. From analysis of evolutionary equations, we obtain the critical values of the kinetic momentum vector, $\vec{L}$, for different values of orbital eccentricity. We find a general tendency of vector $\vec{L}$ to evolve to the direction perpendicular to the orbital plane.
\end{abstract}

Keywords. exoplanets, spin evolution, tidal perturbations

\section{Introduction}

Investigations of the spin motion of celestial bodies were started by d'Alembert, Euler, Lagrange and Laplace. Their results led to the theories of precession and nutation of the Earth's axis and libration of the Moon, and to the general perturbation theory.

Discoveries of extra-solar planets have inspired intensive developments of the planetary dynamics. Unfortunately, the current technical capabilities do not allow us to measure dynamical and physical characteristics of exoplanets, such as the spin period, dynamical flattening, elastic properties, etc. However, the problems of planetary and stellar spins and physical properties of exoplanets can be studied for observed planetary systems through modeling. In particular, the planets in close-in orbits are of great interest for planetary dynamics because of the extreme orbital characteristics, which are quite different from properties of planetary orbits in the Solar System.

In this report, we discuss the spin evolution of hot Jupiters under the action of tidal perturbations. In the next section, we will obtain estimates of the principal moment of inertia of a planet, and calculate the main moments of inertia for nine exoplanets.

\section{Estimates of the principal moment of inertia and dynamical flattening of exoplanets}

The basic properties of evolution of the axis of rotation of a planet, caused by tidal effects are illustrated in Fig. 1. The tidal deformations lead to changes of the planet rotational axis. If the planet has a permanent volume and is liquid with small viscosity, then the tidal bulge is always directed along a line connecting the centers of masses of the star and planet. Otherwise, the tidal bulge lags behind this line by angle $\gamma_{\pi}$ (Fig. 1). For quantitative estimates of tidal perturbations, the dissipative factor, $Q^{-1}=\sin \gamma_{\pi}$, is often used. The deformation of planets may be non-elastic, and, hence, it can be 


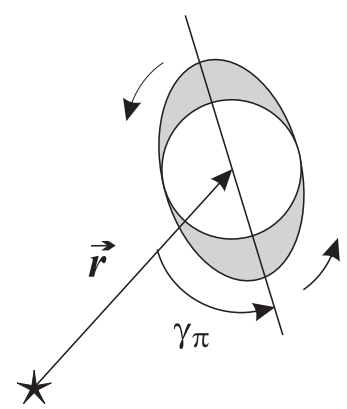

Figure 1. Illustration of the star-planet tidal interaction.

accompanied by significant dissipation of energy. In the case when a central star with mass $M_{\star}$ distorts a spherical exoplanet, dynamic flattening of the planet due to to the tides can be described by the following expression (Alfven \& Arrhenius 1979):

$$
\chi=\frac{15 M_{\star} R^{3}}{4 M_{p} a^{3}},
$$

where $\chi$ is dynamical flattening, $M_{\star}$ and $M_{p}$ are the masses of the star and the planet correspondingly, $R$ is the planetary radius, $a$ is the semi-major axes.

It is obvious that for planets with very close orbits the spin evolution strongly depends on the tidal forces of the parent star. For numerical estimates we consider the spin evolution of nine exoplanets, listed in Table 1. Assuming that the planets are uniform bodies, we calculate the principal momenta of inertia, $I$, and the values of their dynamical flattening, $\chi$. Then using these data we estimate the main moments of inertia $A$ and $B$, shown in Table 2 .

In the next section, we consider the basic equations describing the spin evolution of exoplanets under the action of tidal perturbation of a parent star and calculate the basic evolutionary effects.

Table 1. Some properties of close-in planets.

\begin{tabular}{l|c|c|c|c|c|c|c}
\hline Planet & $\mathrm{M}, M_{J}$ & $\mathrm{R}, R_{J}$ & $a, \mathrm{AU} \mid P$, days & $e$ & $\mathrm{M}, M_{\text {Sun }}$ \\
\hline OGLE-TR-10b & 0.63 & 1.26 & 0.042 & 3.10 & 0 & 1.18 \\
\hline OGLE-TR-56b & 1.29 & 1.3 & 0.023 & 1.212 & 0 & 1.17 \\
\hline OGLE-TR-111 & 0.53 & 1.067 & 0.047 & 4.01 & 0 & 0.82 \\
\hline OGLE-TR-113b & 1.32 & 1.09 & $0.023 \mid$ & 1.43 & 0 & 0.78 \\
\hline OGLE-TR-132b & 1.14 & 1.18 & $0.031 \mid$ & 1.69 & 0 & 1.26 \\
\hline TrES-1b & 0.61 & $1.081 \mid$ & $0.039 \mid$ & 3.03 & $0.135 \mid$ & 0.87 \\
\hline TrES-2b & 1.98 & 1.22 & $0.037 \mid$ & 2.47 & 0 & 0.98 \\
\hline HAT-P-1b & 0.53 & 1.36 & $0.055 \mid$ & 4.47 & 0.09 & 1.12 \\
\hline HD 209458b & 0.69 & 1.32 & $0.045 \mid$ & 3.52 & 0.07 & 1.01 \\
\hline
\end{tabular}




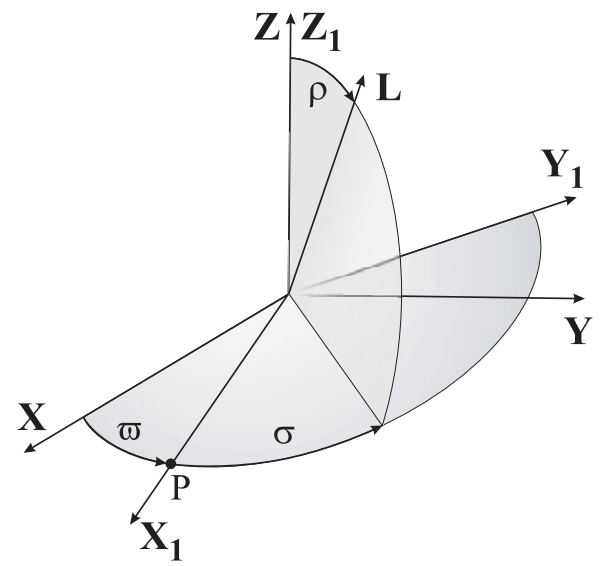

Figure 2. Orientation of kinetic moment vector, $\vec{L}$, relative to the pericentre of the orbit, P.

\section{Spin evolution of planets under action tidal perturbations}

For investigation of the spin evolution of the hot Jupiter planets we use the reference frame, $X_{1} Y_{1} Z_{1}$, which is connected to the orbital pericentre, $P$. In this case, axes $X_{1}$ and $\mathrm{Y}_{1}$ are rotated relative to the orbital reference frame, $\mathrm{XYZ}$, by an angle of the longitude of the pericentre, $\tilde{\omega}$ (Fig. 2). The orientation of the kinetic momentum vector $\vec{L}$ relative to the pericentre of the orbit of a planet is described by angles $\rho$ and $\sigma$.

The perturbed rotation of a planet can be described by the following system of evolutionary equations in the case when the angular spin velocity of a planet is significantly higher than its angular orbital velocity (Beletskii \& Khentov 1995):

$$
\frac{d \rho}{d t}=\frac{1}{L \sin \rho}\left(\frac{\partial U}{\partial \psi} \cos \rho-\frac{\partial U}{\partial \sigma}\right)+\frac{M_{1}}{L}-K_{\Omega} \sin i \cos \left(\sigma+\omega_{\pi}\right),
$$

Table 2. Estimates of the surface gravity, dynamical flattening and the moment of inertia for "hot Jupiters".

\begin{tabular}{|c|c|c|c|c|c|c|}
\hline Planet & $\mid \begin{array}{c}\text { Surface gravity } \\
\mathrm{m} \mathrm{s}^{-2}\end{array}$ & $\chi$ & $\mid \begin{array}{c}I \times 10^{42} \\
\mathrm{~kg} \mathrm{~m} \mathrm{~m}^{2}\end{array}$ & $\begin{array}{c}A \times 10^{42} \\
\mathrm{~kg} \mathrm{~m} \mathrm{~m}^{2}\end{array}$ & $\begin{array}{c}C \times 10^{42} \\
\mathrm{~kg} \mathrm{\textrm {m } ^ { 2 }}\end{array}$ & $\begin{array}{c}F_{\text {tidal }} \times 10^{-10} \\
\mathrm{~N} / \mathrm{kg}\end{array}$ \\
\hline OGLE-TR-10b & 10.07 & 0.020 & 3.79 & 3.82 & 3.74 & 6.49 \\
\hline OGLE-TR-56b & 19.38 & 0.072 & 8.26 & 8.47 & 7.85 & 40.72 \\
\hline OGLE-TR-111 & 11.82 & 0.007 & 2.29 & 2.29 & 2.28 & 3.13 \\
\hline OGLE-TR-113b & 28.20 & 0.026 & 5.94 & 6 & 5.84 & 25.74 \\
\hline OGLE-TR-132b & 20.78 & 0.026 & 6.02 & 6.07 & 5.91 & 17.43 \\
\hline TrES-1b & 13.25 & 0.012 & 2.70 & 2.71 & 2.68 & 5.68 \\
\hline TrES-2b & 33.77 & 0.0075 & 11.17 & 11.2 & 11.11 & 7.86 \\
\hline HAT-P-1b & 7.27 & 0.013 & 3.72 & 3.73 & 3.68 & 2.65 \\
\hline HD $209458 b$ & 10.05 & 0.015 & 4.56 & 4.58 & 4.511 & 4.39 \\
\hline
\end{tabular}

Note:

${ }^{1}$ For comparison: the tidal force of the Jupiter on Io is equal to $9 \times 10^{-37} \mathrm{~N} / \mathrm{kg}$; for the Moon, the tidal action of the Earth is $3.6 \times 10^{-39} \mathrm{~N} / \mathrm{kg}$. 


$$
\begin{aligned}
\frac{d \sigma}{d t}= & \frac{1}{L \sin \rho} \frac{\partial U}{\partial \rho}+\frac{M_{2}}{L \sin \rho}+K_{\Omega}\left(\sin i \cot \rho \sin \left(\sigma+\omega_{\pi}\right)-\cos i\right) \\
\frac{d L}{d t}= & \frac{\partial U}{\partial \psi}+M_{3} \\
\frac{d \vartheta}{d t}= & L \sin \vartheta \sin \phi \cos \phi\left(\frac{1}{A}-\frac{1}{B}\right)+\frac{1}{L \sin \vartheta}\left(\cos \vartheta \frac{\partial U}{\partial \psi}-\frac{\partial U}{\partial \phi}\right)+ \\
& +\frac{M_{2} \cos \psi-M_{1} \sin \psi}{L}, \\
\frac{d \psi}{d t}= & L\left(\frac{\sin ^{2} \phi}{A}+\frac{\cos ^{2} \phi}{B}\right)-\frac{1}{L}\left(\frac{\partial U}{\partial \vartheta} \cot \vartheta+\frac{\partial U}{\partial \rho} \cot \rho\right)- \\
& -\frac{M_{1} \cos \psi+M_{2} \sin \psi}{L} \cot \vartheta-\frac{M_{2}}{L} \cot \rho-K_{\Omega} \frac{\sin i}{\sin \rho} \sin \left(\sigma+\omega_{\pi}\right) \\
\frac{d \phi}{d t}= & L \cos \vartheta\left(\frac{1}{C}-\frac{\sin ^{2} \phi}{A}-\frac{\cos ^{2} \phi}{C}\right)+\frac{1}{L \sin \vartheta} \frac{\partial U}{\partial \vartheta}+ \\
& +\frac{M_{1} \cos \psi+M_{2} \sin \psi}{L \sin \vartheta},
\end{aligned}
$$

where angles $\rho, \sigma$ describe the orientation of vector $\vec{L}$ in the orbital reference frame (Fig. $1) ; \psi, \phi$ and $\vartheta$ are the Euler's angles, $U$ is the potential force function of perturbations, $M_{1}, M_{2}$ and $M_{3}$ are projections of the nonpotential perturbation forces on components of vector $\vec{L}$, and $A, B$ and $C$ are the main moments of inertia of the planet.

In a first approximation we assume that the planet is a dynamically symmetrical body $(A=B \neq C)$ and divide all variables in the system (3.1 - 3.6) into fast and slow. We define fast variables as the variables, which change in the unperturbed motion. The variables, which are constants for the unperturbed motion but vary in the case of perturbations, are called slow variables. Hence, in the case of a dynamically symmetrical planet the slow variables are angles $\rho$ and $\sigma$, which describe position of vector $\vec{L}$ in space (Fig. 2), the value of $\vec{L}$, and the angle of spin rotation, $\phi$. Thus, for the tidal perturbations the spin evolution of "hot Jupiters" can be described by the following dynamical system:

$$
\begin{aligned}
\frac{d \rho}{d t} & =\frac{M_{1}}{L}, \\
\frac{d \sigma}{d t} & =\frac{M_{2}}{L \sin \rho}, \\
\frac{d L}{d t} & =M_{3}, \\
\frac{d \phi}{d t} & =L \cos \vartheta\left(\frac{1}{C}-\frac{1}{A}\right)+\frac{M_{1} \cos \psi+M_{2} \sin \psi}{L \sin \vartheta} .
\end{aligned}
$$

It is known, that if a celestial body has fast non-resonant rotation then the moment of the tidal interaction in the first approximation is described as

$$
\vec{M}=\frac{l_{1} l_{3}}{r^{6}}\left\{\left[\left(\vec{\omega}_{\text {spin }}-\vec{\omega}_{\text {rot }}\right) \times \vec{e}_{r}\right] \times \vec{e}_{r}\right\}
$$

where $\vec{e}_{r}$ is the unit vector of the radius-vector $\vec{r}, l_{1}=1.51 l_{2} \gamma_{g} M_{\star}^{2} R_{p l}^{5}$ is a constant coefficient, $l_{2}$ is a coefficient that depends on elastic properties of the planet (for Jupiter $\left.l_{2}=5 / 2\right)$.

If the tidal perturbations can be described by equation (3.11), then evolutionary 
equations (3.7 - 3.10) become (Beletskii 1981):

$$
\begin{aligned}
\frac{d \rho}{d t} & =\frac{\delta}{2 L P^{6}} \sin \rho\left\{-2 \omega \nu_{1}+\alpha \beta L \cos \rho\left(\nu_{2}+0.5 \nu_{3} \cos 2 \sigma\right)\right\} \\
\frac{d \sigma}{d t} & =-\frac{\delta}{4 P^{6}} \alpha \beta \nu_{3} \sin 2 \sigma \\
\frac{d L}{d t} & =\frac{\delta}{P^{6}}\left\{\omega \nu_{1} \cos \rho-\alpha \beta L\left(\nu_{2}\left(1-0.5 \sin ^{2} \rho\right)-0.5 \nu_{3} \sin ^{2} \rho \cos 2 \sigma\right)\right\} \\
\frac{d \phi}{d t} & =L \cos \vartheta\left(\frac{1}{C}-\frac{1}{A}\right)
\end{aligned}
$$

where $\alpha=\sin ^{2} \vartheta / A+\cos ^{2} \vartheta / C, \beta=\left(1-e^{2}\right)^{3 / 2}, P$ is the semilatus rectum, $\delta=l_{1} l_{3}$, $\omega=\sqrt{G M_{\star} / a^{3}}$ is the angular velocity of the orbital motion, $G$ is the gravity constant, $e$ is eccentricity, $\nu_{1}=1+7.5 e^{2}+5.625 e^{4}+\ldots, \nu_{2}=1+3 e^{2}+0.375 e^{4}, \nu_{3}=1.5 e^{2}+0.25 e^{4}$.

Equation (3.15) that describes evolution of the angle of the planet's spin $\phi$, depends only on the vector of kinetic moment $\vec{L}$ of a planet and the values of the main moments of inertia (we assume that the angle of nutation, $\vartheta$, is constant). Consequently we have only three independent equations (3.12 - 3.14). In addition, equation (3.13) is independent from the other two. Therefore, we can reduce the evolutionary equations to a system of two equations $(3.12,3.14)$. After the change of variables:

$$
\begin{array}{lll}
x=\rho, & y=L, & \dot{x}=d \rho / d t, \quad \dot{y}=d L / d t, \\
a=\delta /\left(2 P^{6}\right), & b=\omega \nu_{1}, & c=\alpha \beta, \quad d=\nu_{2}+0.5 \nu_{3} \cos 2 \sigma
\end{array}
$$

we obtain the following system:

$$
\begin{aligned}
& \dot{x}=\frac{a}{y} \sin \rho(-2 b+c d y \cos x), \\
& \dot{y}=2 a\left[b \cos x-0.5 c y\left(\nu_{2}\left(1+\cos ^{2} x\right)-\nu_{3} \cos 2 \sigma \sin ^{2} x\right)\right] .
\end{aligned}
$$

We investigate stability of the dynamic system (3.16) from the point of view the Lyapunov's stability by methods of the qualitative analysis (Kitiashvili \& Gusev 2008). The dynamical system (3.16) has six equilibrium states (ESs) with coordinates:

$$
\begin{aligned}
x_{1,2} & =0, \pi, & y_{1,2} & = \pm \frac{b}{c \nu_{2}} \\
x_{3,4} & = \pm \arccos \xi, & y_{3,4} & =\frac{2 b}{c d} \xi \\
x_{5,6} & = \pm \arccos (-\xi), & y_{3,4} & =-\frac{2 b}{c d} \xi,
\end{aligned}
$$

where

$$
\xi=\sqrt{\frac{0.5 \nu_{3} \cos 2 \sigma}{\nu_{3} \cos 2 \sigma-\nu_{2}}}
$$

Table 3. Critical values of parameters for planets at close-in orbits $(e=0)$.

\begin{tabular}{l|c|c|c}
\hline Planet & ES 1-2: L for $\rho=0^{0}, 180^{0}$ & Planet & $\mid$ ES $1-2:$ L for $\rho=0^{0}, 180^{0}$ \\
\hline OGLE-TR-10b & $1.39 \times 10^{37}$ & $\mid$ OGLE-TR-113b & $4.72 \times 10^{37}$ \\
\hline OGLE-TR-56b & $7.51 \times 10^{37}$ & $\mid$ OGLE-TR-132b & $4.05 \times 10^{37}$ \\
\hline OGLE-TR-111 & $6.6 \times 10^{36}$ & $\mid$ TrES-2b & $5.21 \times 10^{37}$ \\
\hline
\end{tabular}


Table 4. Critical values of parameters for planets with $e \neq 0$.

\begin{tabular}{l|c|c|c|c|c}
\hline Planet & $\begin{array}{c}\text { ES 1-2: } \\
L \times 10^{37}\end{array}$ & $\begin{array}{c}\text { ES 3-4: } \\
\rho\end{array}$ & $\begin{array}{c}\text { ES 3-4: } \\
L \times 10^{35}\end{array}$ & $\begin{array}{c}\text { ES 5-6: } \\
\rho\end{array}$ & $\begin{array}{c}\text { ES 5-6: } \\
L \times 10^{35}\end{array}$ \\
\hline HD 209458b & 1.52 & $\pm\left(1.56^{0}-1.53^{0}\right)$ & $3.14-13.08$ & $\pm\left(1.58^{0}-1.61^{0}\right)$ & $3.14-13.08$ \\
\hline HAT-P-1b & 1.00 & $\pm\left(1.56^{0}-1.52^{0}\right)$ & $2.64-10.99$ & $\pm\left(1.58^{0}-1.63^{0}\right)$ & $2.64-10.99$ \\
\hline TrES-1b & 1.14 & $\pm\left(1.56^{0}-1.49^{0}\right)$ & $4.43-18.45$ & $\pm\left(1.59^{0}-1.65^{0}\right)$ & $4.43-18.45$ \\
\hline
\end{tabular}

The six equilibrium states $(3.17-3.19)$ are possible only at $e \neq 0$. In the case of a circular orbit, there are only two ESs. Numerical estimates of angle $\rho$ and the value of the kinetic moment $\vec{L}$ for the ESs are presented in Table 3 for the planets with $e=0$, and in Table 4 for the planets with $e \neq 0$. The phase trajectories in the vicinity of ES 1 and 2 are of the steady node type, while the trajectories for other ES have the type of steady focus. Thus, the value of $\vec{L}$ evolves to the value of $b /\left(c \nu_{2}\right)\left(\sim 1.14 \times 10^{37}\right)$ or $2 b \xi /(c d)\left(\sim 4.43 \times 10^{35}\right.$ for TrES-1b in Table 4 . The orientation of the kinetic moment vector, $\vec{L}$, tend to evolve to the direction perpendicular to the orbital plane of a planet, $\rho=0^{0}$ or $\rho \sim 1.6^{0}$ for TrES-1b. In Table 4 , we show the numerical estimates of the critical values of angle $\rho$ and $\vec{L}$. Possible variations of the $\rho$ and $\vec{L}$ values, which depend on the assumed angle $\sigma$, are shown in the brackets.

According to our results, under the action of the tidal forces between a hot Jupiter planet and a parent star the vector of the kinetic moment $\vec{L}$ of the planet evolves to the position perpendicular to the orbital plane in the case of a circular orbit or close to this position for elliptical orbits.

\section{Conclusions}

In this report, we have considered possible scenarios of the spin evolution of exoplanets at close orbits. We have found the effect of the evolution of orientation of the exoplanet spin to the position perpendicular to the orbital plane for circular orbits or close to this orientation in the case of elliptical orbits. We have obtained the dynamical estimates of the principal moment of inertia of some hot Jupiter planets, and their dynamical flattening under the action of tidal forces from the parent stars.

\section{References}

Alfven, H., \& Arrhenius, G. 1976, Evolution of the Solar system. Scientific and technical information office NASA, Washindton, D.C., USA.

Beletskii, V.V. 1981, Celestial Mechanics, 23, 371.

Beletsky, V.V. \& Khentov, A.A. 1995 Resonance rotation of celestial bodies. Nizhnii Novgorod, Nizhegorodskii gumanitarnii centr, Russia (in Russian).

Kitiashvili, I. \& Gusev, A. 2008, Celestial Mecanics and Dynamical Astronomy, 100 (in press). 